\title{
Reprocessing the Hipparcos data for evolved giant stars II. Absolute magnitudes for the R-type carbon stars ${ }^{\star}$
}

\author{
G. Knapp ${ }^{1}$, D. Pourbaix ${ }^{1,2, \star \star}$, and A. Jorissen ${ }^{2, \star \star \star}$ \\ 1 Department of Astrophysical Sciences, Princeton University, Princeton, NJ 08544-1001, USA \\ 2 Institut d'Astronomie et d'Astrophysique, Université Libre de Bruxelles, CP 226, Boulevard du Triomphe, \\ 1050 Bruxelles, Belgium
}

Received 9 August 2000 / Accepted 8 March 2001

\begin{abstract}
The Hipparcos Intermediate Astrometric Data for carbon stars have been reprocessed using an algorithm which provides an objective criterion for rejecting anomalous data points and constrains the parallax to be positive. New parallax solutions have been derived for 317 cool carbon stars, mostly of types $\mathrm{R}$ and N. In this paper we discuss the results for the $\mathrm{R}$ stars. The most important result is that the early $\mathrm{R}$ stars (i.e., R0 - R3) have absolute magnitudes and $V-K$ colors locating them among red clump giants in the Hertzsprung-Russell diagram. The average absolute magnitude $M_{\mathrm{K}}$ for early R-type stars (with $V-K<4$ ) has been derived from a Monte-Carlo simulation implicitly incorporating all possible biases. It appears that the simulated magnitude distribution for a population with a true Gaussian distribution of mean $M_{K}=-2.0$ and intrinsic standard deviation $1.0 \mathrm{mag}$ provides a satisfactory match to the observed distribution. These values are consistent with the average absolute magnitude $M_{\mathrm{K}}=-1.6$ for clump red giants in the solar neighborhood (Alves 2000). Further, early R-type stars are non-variable, and their infrared photometric properties show that they are not undergoing mass loss, properties similar to those of the red clump giants. Stars with subtypes R4- R9 tend to be cooler and have similar luminosity to the N-type carbon stars, as confirmed by their position in the $(J-H, H-K)$ color-color diagram. The sample of early R-type stars selected from the Hipparcos Catalogue appears to be approximately complete to magnitude $K_{0} \sim 7$, translating into a completeness distance of $600 \mathrm{pc}$ if all $\mathrm{R}$ stars had $M_{K}=-2\left(400 \mathrm{pc}\right.$ if $\left.M_{K}=-1\right)$. With about 30 early R-type stars in that volume, they comprise about $0.04 \%\left(0.14 \%\right.$ for $\left.M_{K}=-1\right)$ of the red clump stars in the solar neighborhood. Identification with the red clump locates these stars at the helium core burning stage of stellar evolution, while the $\mathrm{N}$ stars are on the asymptotic giant branch, where helium shell burning occurs. The present analysis suggests that for a small fraction of the helium core burning stars (far lower than the fraction of helium shell-burning stars), carbon produced in the interior is mixed to the atmosphere in sufficient quantities to form a carbon star.
\end{abstract}

Key words. stars: carbon - stars: horizontal branch - astrometry - stars: distance - Hertzsprung-Russell diagram

\section{Introduction}

The Harvard spectroscopic classification of stars for the Henry Draper Catalogue recognized the first examples of chemically-peculiar stars, the carbon and $\mathrm{S}$ giants. Carbon stars are immediately recognizable by the presence of absorption bands of $\mathrm{C}_{2}$ at $4383 \AA$ and $4737 \AA$ and the $4216 \AA$ band of CN (Shane 1928), and are chemically characterized by having $\mathrm{C} / \mathrm{O}>1$. Pickering $(1896,1908)$ showed that the carbon stars further subdivide into two spectral

\footnotetext{
Send offprint requests to: G. Knapp,

e-mail: gk@astro.princeton.edu

* Based on observations from the Hipparcos astrometric satellite operated by the European Space Agency (ESA 1997).

${ }_{\star \star \star}$ Post-doctoral Researcher, F.N.R.S., Belgium.

$\star \star \star$ Research Associate, F.N.R.S., Belgium.
}

classes, the "warm" R stars and the "cool" N stars, and in the intervening years it has become increasingly apparent that these stars do not represent a continuum of properties but rather belong to two distinct classes. Further, Keenan (1942) noted that some members of the R spectral class were metal-poor and had high radial velocities and proper motions. These are now separately classified as CH stars. Several low-concentration globular clusters contain CH stars (Bond 1975; McClure 1984b); they are generally considered to be halo carbon stars. The most recent list of CH stars is by Bartkevičius (1996). Several additional types of carbon star have been recognized, including $\mathrm{R}$ CrB stars and dwarf carbon stars.

The revised classification system of Keenan (1993) is an attempt to provide consistent temperature, luminosity and abundance sequences among the different types of 
carbon stars. Three main classes are defined in this scheme: C-R $n, \mathrm{C}-\mathrm{N} n$, and $\mathrm{C}-\mathrm{H} n$, corresponding to the old $\mathrm{R}, \mathrm{N}$ and $\mathrm{CH}$ types respectively, with $n$ being a homogeneous temperature index for the three sequences, corresponding to the G-K-M temperature sequence in "oxygen" stars. Unfortunately, spectral types based on this scheme are available for only a small fraction of carbon stars.

Since $\mathrm{C} / \mathrm{O}<1$ everywhere in the interstellar medium, in the Sun, and in most stars, the excess carbon in carbon stars must be the result of stellar nucleosynthesis in the star itself or in a binary companion. The many varieties of carbon stars are thus stars in the late stages of evolution or with evolved binary companions, and are preferentially formed in systems whose intrinsic metallicities (including the oxygen abundance) are low.

The N stars are understood in a general sense. They are very luminous, cool stars with shell hydrogen and helium burning and with $3 \alpha$-produced ${ }^{12} \mathrm{C}$ mixed to the surface by deep convection (Iben \& Renzini 1983), although the details of how that mixing occurs are far from well understood. Other stellar types with enhanced carbon abundance, like $\mathrm{CH}$ and barium stars, are observed to be members of binary systems (see the discussions by McClure 1984a, 1997a; Jorissen et al. 1998), and their peculiar abundances can be attributed to mass transfer. The $\mathrm{R}$ $\mathrm{CrB}$ stars are variable hydrogen-deficient stars, and have shed most of their envelope. The dwarf carbon stars (Dahn et al. 1977; Green \& Margon 1994) and the R stars remain a mystery. Like the $\mathrm{N}$ stars, the $\mathrm{R}$ stars are red giants, but their average luminosity, determined by statistical parallax methods (Vandervoort 1958; Scalo 1976; Dean 1976) is too low for shell helium burning. Further, their oxygen abundances are similar to that of the Sun (Dominy 1984), they do not exhibit $s$-process overabundances, and no $\mathrm{R}$ star has been found so far in a binary system (McClure 1997b), a statistically unlikely and surprising result. It has therefore been suggested that $\mathrm{R}$ stars might actually be coalesced binary systems. The remaining hypothesis is that these stars are helium core-burning stars or post helium core burning stars, with carbon produced by helium burning somehow introduced into their atmospheres, perhaps at the helium core flash (Scalo 1976; Dominy 1984). There is little direct evidence for this hypothesis, though, because of lack of information about the intrinsic luminosity function of the $\mathrm{R}$ stars or of their spatial density. This paper sets out to derive the luminosities of the $\mathrm{R}$ stars to attempt to discover their location on the HertzsprungRussell diagram and establish their evolutionary status.

The Hipparcos project (ESA 1997) made it possible for the first time to make direct estimates of the distances to giant carbon stars. However, as noted by Knapp (2001), the parallax errors for these stars are often far larger than expected. In this paper, we use new parallaxes derived from a re-reduction of the Hipparcos Intermediate Astrometric Data to show that essentially all the (early) R stars have the same absolute magnitude and color, and are located in the red clump region of the HR diagram. The Hipparcos parallaxes also allow us to derive the spatial density of the red clump stars and the fraction of these which are $\mathrm{R}$ stars; we show that they are very rare, far less than $1 \%$ of the red clump stars (Sect. 4). The carbon enhancements in R stars may therefore originate in the helium-burning interiors, perhaps as the result of a mixing in a rapidly rotating star spun up by the accretion of its companion (see Sect. 5). Section 2 discusses the astrometric, photometric, spectroscopic and variability data on which the results presented in this paper are based. The absolute magnitudes and colors are examined in Sect. 3, the space densities in Sect. 4, and the implications and conclusions are discussed in Sect. 5. The details of the re-reduction of the Hipparcos data will be discussed by Pourbaix et al. (2001a, Paper III) and the results for N-type carbon stars by Pourbaix et al. (2001b, Paper IV).

\section{The data}

\subsection{Overview}

The basic data for $\mathrm{R}$ stars, including the new parallax data, are collected in Tables 1 and 2. Stars with enhanced carbon abundances come in many spectral types: the $\mathrm{N}$ stars, the $\mathrm{R}$ stars, carbon white dwarfs, carbonrich Wolf-Rayet stars, carbon cepheids, $\mathrm{R}$ CrB stars, barium stars, CH stars, and dwarf carbon stars, among others. As pointed out above, their chemical peculiarities are due to nucleosynthesis within the star itself, or its companion, and the task is to identify chemical peculiarities with particular evolutionary paths. This is carried out in the first instance by associating like objects, classifying them by spectral type, luminosity, color, and variability, and is made difficult both by the wide variety of stars with carbon enhancements, and by the often uncertain observational information. A given star can be classified as several different spectral types in several different papers, causing confusion in attempts to evaluate the common properties of given classes of stars (see the discussions by McClure 1997a,b; Wallerstein \& Knapp 1998). Examples include HIP 12028 (HD 161115) which is classified as R by Vandervoort (1958), as CH by Keenan (1993) and as J by Ohnaka \& Tsuji (1999), while HIP 85750 $\left(\mathrm{BD}+02^{\circ} 3336\right)$ is classified as R2 by Vandervoort (1958), as CH by Yamashita (1975), and as N by Barnbaum et al. (1996). Thus it is far from straightforward to decide, from the available data, whether the presence of a star in a given variability class with a spectral type atypical of that class demonstrates a range of spectral types in that variability class or is due to inadequate data. The infrared color-color diagram $(J-H, H-K)$ (Fig. 1) may nevertheless be of some help in the process of identifying misclassified stars, as the different types of carbon stars occupy specific locations in that diagram (see Sect. 2.2).

Of the many varieties of carbon star, the cool luminous carbon stars are of interest because they are expelling carbon-enhanced mass into the interstellar medium. Knapp (2001) identified about 320 carbon stars in the Hipparcos catalogue. An attempt was made to be 
Table 1. Early R stars: astrometric, photometric and spectroscopic data. The rederived parallax $\varpi_{\circ}$ and its lower and upper bounds (respectively $\varpi_{\mathrm{L}}$ and $\varpi_{\mathrm{U}}$; see text) are given in milliarcseconds (mas). The $\mathrm{R}$ stars have been separated into early and late types, according to the $V-K$ color index being smaller or larger than 4 (see Fig. 3 )

\begin{tabular}{|c|c|c|c|c|c|c|c|c|c|c|c|c|}
\hline HIP & GCGCS & $\begin{array}{l}\varpi_{L} \\
\text { (mas) }\end{array}$ & $\begin{array}{l}\varpi_{\circ} \\
\text { (mas) }\end{array}$ & $\begin{array}{l}\varpi_{\mathrm{U}} \\
(\mathrm{mas})\end{array}$ & $V$ & $K$ & $J-H$ & $H-K$ & $\begin{array}{l}\text { Ref } \\
J H K\end{array}$ & $\begin{array}{l}\text { Sp. } \\
\text { type }\end{array}$ & $\begin{array}{l}\text { Ref } \\
\text { SpT }\end{array}$ & Rem. \\
\hline 2700 & 80 & 1.32 & 2.37 & 4.24 & 9.62 & 6.94 & 0.51 & 0.16 & D86 & $\mathrm{R} 2$ & B96 & \\
\hline 5809 & 196 & 0.24 & 1.09 & 5.05 & 10.07 & 7.22 & 0.77 & 0.03 & C79 & R0 & HD & \\
\hline 7816 & 256 & - & - & - & 11.11 & 7.90 & 1.02 & 0.17 & D86 & R0 & S69 & \\
\hline 11508 & 357 & 0.25 & 1.39 & 3.78 & 9.57 & 5.92 & - & - & D86 & $\mathrm{R} 0$ & V58 & \\
\hline 12028 & 378 & 3.52 & 4.57 & 5.93 & 8.15 & 5.38 & 0.62 & 0.15 & F92 & $\mathrm{R} 2$ & V58 & \\
\hline 17933 & 576 & 0.54 & 1.11 & 2.29 & 8.29 & 4.65 & 0.73 & 0.19 & C81 & $\mathrm{R} 3$ & HD & \\
\hline 18564 & 588 & 0.37 & 1.49 & 4.51 & 10.26 & 6.66 & 0.41 & 0.23 & D86 & R3 & S28 & \\
\hline 18696 & 594 & - & - & - & 10.45 & 7.23 & 0.58 & 0.15 & $2 \mathrm{M}$ & $\mathrm{R} 2$ & S28 & \\
\hline 19050 & 610 & - & - & - & 10.71 & 8.10 & 0.48 & 0.16 & $2 \mathrm{M}$ & $\mathrm{R} 0$ & V58 & \\
\hline 19269 & 639 & 0.72 & 1.74 & 4.20 & 10.62 & 6.97 & 0.56 & 0.46 & C79 & R3 & HD & \\
\hline 26927 & 1035 & 0.17 & 1.08 & 6.57 & 10.02 & 7.62 & 0.5 & 0.09 & D86 & R0 & V58 & \\
\hline 28172 & 1110 & 0.27 & 1.33 & 4.40 & 10.35 & 7.62 & 0.53 & -0.11 & D86 & $\mathrm{R} 2$ & S28 & \\
\hline 31725 & 1380 & 0.46 & 1.10 & 2.66 & 9.41 & 6.46 & 0.54 & 0.21 & C79 & R0 & HD & \\
\hline 33042 & 1460 & - & - & - & 10.56 & - & - & - & - & $\mathrm{R}$ & S72 & \\
\hline 35681 & 1622 & 0.37 & 1.05 & 2.99 & 8.52 & 6.16 & 0.4 & 0.18 & $2 \mathrm{M}$ & $\mathrm{R} 0$ & V58 & $*$ \\
\hline 36086 & 1703 & 0.13 & 1.08 & 4.48 & 9.22 & 5.81 & 0.61 & 0.34 & D86 & R3 & S28 & \\
\hline 39118 & 1981 & 0.06 & 1.32 & 7.23 & 10.41 & 7.23 & 0.79 & 0.25 & $2 \mathrm{M}$ & $\mathrm{R} 2$ & V58 & \\
\hline 40374 & 2126 & - & - & - & 11.70 & 8.38 & 0.6 & 0.22 & $2 \mathrm{M}$ & $\mathrm{R}$ & GCGCS & \\
\hline 44172 & 2396 & 0.81 & 2.08 & 5.34 & 9.44 & 6.84 & - & - & D86 & $\mathrm{R} 2$ & B96 & \\
\hline 48329 & 2626 & 0.35 & 1.22 & 4.23 & 9.90 & 6.25 & 0.7 & 0.22 & D86 & $\mathrm{R} 3$ & S28 & \\
\hline 50994 & 2759 & 0.35 & 1.05 & 3.14 & 9.56 & - & - & - & - & $\mathrm{R} 0$ & HD & \\
\hline 53354 & 2892 & - & - & - & 10.54 & 7.62 & 0.61 & 0.17 & $2 \mathrm{M}$ & $\mathrm{R} 2$ & V58 & \\
\hline 53522 & 2900 & 0.63 & 1.81 & 5.15 & 10.14 & - & - & - & - & $\mathrm{R} 0$ & V58 & \\
\hline 53810 & 2925 & - & - & - & 8.35 & - & - & - & - & K5R & S44 & \\
\hline 53832 & 2919 & 0.96 & 2.08 & 4.52 & 10.11 & - & - & - & - & $\mathrm{R} 0$ & V58 & \\
\hline 56405 & 3058 & 0.12 & 1.19 & 6.52 & 10.27 & - & - & - & - & $\mathrm{R} 2$ & S28 & \\
\hline 58786 & 3156 & 0.26 & 1.06 & 4.20 & 10.27 & 7.51 & 0.52 & 0.15 & $2 \mathrm{M}$ & $\mathrm{R} 2$ & V58 & \\
\hline 62944 & & 7.41 & 8.22 & 9.12 & 6.91 & 4.16 & 0.55 & 0.1 & E78 & $\mathrm{R} 3$ & B96 & \\
\hline 63955 & 3335 & 1.44 & 2.26 & 3.54 & 8.50 & 5.97 & 0.46 & 0.18 & $2 \mathrm{M}$ & $\mathrm{R} 3$ & B96 & \\
\hline 65320 & 3379 & 1.84 & 2.94 & 4.71 & 9.66 & - & - & - & - & $\mathrm{R} 0$ & S28 & \\
\hline 66317 & 3409 & - & - & - & 12.39 & - & - & - & - & $\mathrm{R}$ & S72 & \\
\hline 68543 & 3469 & 0.21 & 1.02 & 5.07 & 9.48 & 6.67 & 0.52 & 0.19 & D86 & $\mathrm{R} 2$ & S28 & \\
\hline 69089 & & 0.53 & 1.17 & 2.59 & 8.68 & 6.40 & 0.46 & 0.12 & $2 \mathrm{M}$ & $\mathrm{R} 2$ & B96 & \\
\hline 71464 & 3513 & - & - & - & 12.17 & - & - & - & - & $\mathrm{R}$ & S72 & \\
\hline 73955 & 3591 & 0.42 & 1.17 & 3.28 & 9.99 & - & - & - & - & R0 & S28 & \\
\hline 74179 & 3562 & 0.28 & 1.16 & 4.83 & 9.72 & 6.50 & 0.5 & 0.5 & F97 & $\mathrm{R} 3$ & HD & $*$ \\
\hline 74826 & 3586 & 0.52 & 1.55 & 4.64 & 9.78 & - & - & - & - & $\mathrm{R} 0$ & S28 & \\
\hline 75691 & 3614 & - & - & - & 12.88 & 11.12 & 0.4 & 0.06 & $2 \mathrm{M}$ & $\mathrm{R} 5$ & V58 & $*$ \\
\hline 75745 & 3605 & - & - & - & 11.79 & - & - & - & - & $\mathrm{R}$ & S72 & \\
\hline 80365 & 3687 & - & - & - & 11.18 & 8.16 & 0.23 & 0.5 & F73 & $\mathrm{R}$ & M51 & $*$ \\
\hline 80769 & 3704 & 0.37 & 1.37 & 5.11 & 10.40 & 7.50 & - & - & M65 & $\mathrm{R} 2$ & V58 & \\
\hline 82184 & 3735 & 0.76 & 1.74 & 3.99 & 9.10 & 6.46 & 0.57 & 0.12 & $2 \mathrm{M}$ & $\mathrm{R} 2$ & B96 & \\
\hline 84266 & 3795 & 2.30 & 2.89 & 3.62 & 7.60 & 5.11 & 0.3 & 0.23 & N81 & $\mathrm{R} 2$ & B96 & \\
\hline 85117 & 3816 & 1.88 & 3.33 & 5.91 & 9.57 & - & - & - & - & $\mathrm{R} 3$ & $\mathrm{C} 21$ & \\
\hline 86927 & 3879 & 3.77 & 4.78 & 6.05 & 8.71 & 6.14 & 0.53 & 0.14 & D86 & R0 & $\mathrm{S} 28$ & \\
\hline 87603 & 3912 & 0.30 & 1.05 & 3.66 & 10.72 & 7.92 & 0.55 & 0.18 & $2 \mathrm{M}$ & $\mathrm{R} 4$ & S44 & \\
\hline 88584 & 3938 & 2.07 & 3.49 & 5.88 & 9.82 & - & - & - & - & $\mathrm{R} 3$ & S44 & $*$ \\
\hline 89239 & 3973 & 4.56 & 6.37 & 8.91 & 10.82 & 7.41 & 0.61 & 0.24 & $2 \mathrm{M}$ & $\mathrm{R} 5$ & V58 & \\
\hline
\end{tabular}

complete (as far as the members of the Hipparcos catalogue are concerned) for the luminous cool carbon stars, i.e., the $\mathrm{R}$ and $\mathrm{N}$ stars (because these are the stars which are, or may be, losing mass). Although the Hipparcos catalogue itself is based only partly on a complete input catalogue (the Input Catalogue contained both complete samples of stars - designed as "Hipparcos survey stars" - and additional samples of stars of particular interest), it turns out to be reasonably complete for carbon stars

${ }^{1}$ Only 3 such stars (HIP 12028, HIP 62944 and HIP 84266 discussed herein) are present in the sample of $\mathrm{R}$ stars. 
Table 1. continued

\begin{tabular}{|c|c|c|c|c|c|c|c|c|c|c|c|c|}
\hline HIP & GCGCS & $\begin{array}{l}\varpi_{\mathrm{L}} \\
\text { (mas) }\end{array}$ & $\begin{array}{l}\varpi_{\circ} \\
\text { (mas) }\end{array}$ & $\begin{array}{l}\varpi_{\mathrm{U}} \\
\text { (mas) }\end{array}$ & $V$ & $K$ & $J-H$ & $H-K$ & $\begin{array}{l}\text { Ref } \\
J H K\end{array}$ & $\begin{array}{l}\text { Sp. } \\
\text { type }\end{array}$ & $\begin{array}{l}\text { Ref } \\
\text { SpT }\end{array}$ & Rem. \\
\hline 89739 & 3982 & 2.53 & 4.61 & 8.43 & 10.67 & 8.00 & 0.5 & 0.5 & F97 & R0 & $\mathrm{C} 21$ & $*$ \\
\hline 90199 & 4002 & - & - & - & 11.91 & - & - & - & - & $\mathrm{R} 0$ & S44 & \\
\hline 90694 & 4021 & 0.15 & 1.19 & 5.56 & 9.88 & 6.30 & 0.67 & 0.23 & C79 & $\mathrm{R} 5$ & S44 & \\
\hline 92207 & 4098 & 0.26 & 1.62 & 6.91 & 10.00 & 6.5 & 1 & 1 & F97 & R0 & $\mathrm{HD}$ & $*$ \\
\hline 93987 & 4181 & 0.33 & 2.09 & 13.40 & 11.08 & 5.89 & 0.54 & 0.52 & $2 \mathrm{M}$ & $\mathrm{R} 2$ & $\mathrm{~S} 44$ & $*$ \\
\hline 95392 & 4485 & 0.39 & 1.06 & 2.86 & 9.66 & - & - & - & - & $\mathrm{R} 2$ & $\mathrm{~S} 28$ & \\
\hline 98057 & 4560 & 0.24 & 1.12 & 5.23 & 9.62 & - & - & - & - & R0 & S28 & \\
\hline 99725 & 4784 & 0.48 & 1.10 & 2.49 & 9.75 & 6.05 & 0.7 & 0.15 & U97 & R0 & V58 & \\
\hline 102726 & 4972 & - & - & - & 10.64 & - & - & - & - & $\mathrm{R} 1$ & S44 & \\
\hline 105212 & 5227 & 0.98 & 2.31 & 5.44 & 9.71 & - & - & - & - & $\mathrm{R} 2$ & V58 & \\
\hline 105241 & 5230 & 1.56 & 3.11 & 6.19 & 9.81 & 6.97 & 0.5 & 0.15 & D86 & $\mathrm{R} 2$ & V58 & \\
\hline 107349 & 5408 & 0.20 & 1.04 & 5.42 & 10.16 & 5.51 & 0.91 & 0.36 & C79 & R0 & HD & \\
\hline 114452 & 5822 & 0.72 & 1.70 & 4.00 & 9.99 & 7.03 & 0.47 & 0.2 & D86 & $\mathrm{R} 2$ & $\mathrm{~S} 28$ & \\
\hline 114509 & 5823 & 1.92 & 3.24 & 5.47 & 9.30 & 6.69 & 0.96 & 0.11 & D86 & R0 & S44 & \\
\hline 117467 & 5937 & 0.98 & 1.97 & 3.95 & 8.52 & 5.42 & 0.61 & 0.21 & C79 & R3 & B96 & \\
\hline
\end{tabular}

\section{Notes:}

HIP 35681: RU Cam, type CWa, period 22 d; HIP 74179: S Aps, type R CrB; HIP 75691: NSV 7110; HIP 80365: RT Nor, type R CrB; HIP 88584: W CrA, type SRb, period 125 d; HIP 89739: RS Tel, type R CrB; HIP 92207: V CrA, type R CrB; HIP 93987: SV Sge, type R CrB.

\section{References to spectral types and magnitudes:}

HD: Henry Draper Catalogue; GCGCS: Stephenson (1989); 2M: Two Micron All-Sky Survey (Skrutskie 1997); B96: Barnbaum et al. (1996); C21: Cannon (1921); C79: Catchpole et al. (1979); C81: Cohen et al. (1981); D86: Dominy et al. (1986); E78: Elias (1978); F73: Feast \& Glass (1973); F92: Feast \& Whitelock (1992); F97: Feast et al. (1997); M51: Mayall (1951); M65: Mendoza V. \& Johnson (1965); N81: Noguchi et al. (1981); S28: Shane (1928); S44: Sanford (1944); S69: Slettebak et al. (1969); S72: Stock \& Wroblewski (1972); U97: Ulla et al. (1997); V58: Vandervoort (1958).

to Hipparcos magnitude $H p \sim 9.5$ (Knapp 2001) or to $K \sim 7$ (Fig. 6).

\subsection{Photometry}

We use the $V$ magnitude given in the Hipparcos catalogue (field $\mathrm{H} 5$ ). We acquired $J, H$ and $K$ photometry from the literature, as listed in Tables 1 and 2 along with the bibliographic reference. This was found with the aid of the electronic version of the literature compilation catalogue of Gezari et al. (1999). In addition, we extracted $K_{\mathrm{s}}$ photometry from the Two Micron All Sky Survey (2MASS) on-line data release (Skrutskie 1997). In many cases, the stars are bright enough that they are saturated in the 2MASS data and we use older photometry. There are no systematic offsets evident among the various photometric measures for a given star, and the measurements (including the narrower 2MASS $K_{\mathrm{S}}$ band magnitudes) agree to better than about $0.2 \mathrm{mag}$ in almost all cases. We were able to find $K$ magnitudes for all but 24 stars. Although these observations were made at different epochs, $\mathrm{R}$ stars are not in general variable (Sect. 2.3) and so there is little or no uncertainty introduced into the $V$ or $K$ magnitudes by variability.

Figure 1 presents the $(J-H, H-K)$ color-color diagram. It is immediately apparent that $\mathrm{R}$ stars occupy a very specific region of that diagram, which is also populated by $\mathrm{CH}$ stars. A few $\mathrm{R}$ stars appear to occupy the same region as N-type carbon stars, which is not surprising as most - if not all - late R-type carbon stars may actually be $\mathrm{N}$ stars (see the discussion in Sects. 2.3 and 2.4).

\subsection{Variability}

$\mathrm{N}$ stars are in general long-period variables: irregular (Lb), semi-regular (SR or SRb) or Mira (Mira or SRa) - see Kerschbaum \& Hron (1994) and Wallerstein \& Knapp (1998) for a discussion of the SRa and SRb stars. R stars generally do not appear in variable star catalogues. Of the $83 \mathrm{R}$ stars in our sample, 18 appear in the General Catalogue of Variable Stars (Kholopov et al. 1998), but most of these are late $\mathrm{R}$ stars, as listed in Table 2. Most of the R stars of variability types Mira and SR are also losing mass, as indicated by their excess $12 \mu \mathrm{m}$ emission (Table 2), and are therefore probably closely related to $\mathrm{N}$ stars.

\subsection{Spectral types}

There are two major studies of $\mathrm{R}$ stars. Vandervoort (1958) observed a sample of $98 \mathrm{R}$ stars selected from an objective prism survey. McClure (1997b) discusses a sample of $22 \mathrm{R}$ stars. We have also used spectral types from Keenan (1993), Barnbaum et al. (1996), and other references. In toto, we have a sample of $83 \mathrm{R}$ stars. The data 
Table 2. Late R stars: astrometric, photometric and spectroscopic data. Columns have the same meaning as in Table 1. Additional columns provide the variability name, type and period (from GCVS), and the $K-[12](=K+2.5 \log F 12 / 28.3)$ color index (where $F 12$ is the flux density in the IRAS $12 \mu \mathrm{m}$ band expressed in Jy). The $K-[12]$ index is a mass-loss indicator, non-mass-losing stars having $K-[12] \sim 0.7$ (e.g. Jorissen \& Knapp 1998)

\begin{tabular}{|c|c|c|c|c|c|c|c|c|c|c|c|c|c|c|c|c|}
\hline$\overline{\mathrm{HIP}}$ & GCGCS & $\begin{array}{l}\varpi_{\mathrm{L}} \\
\text { (mas) }\end{array}$ & $\begin{array}{l}\varpi_{\circ} \\
\text { (mas) }\end{array}$ & $\begin{array}{l}\varpi_{\mathrm{U}} \\
\text { (mas) }\end{array}$ & $V$ & $K$ & $J-H$ & $H-K$ & $\begin{array}{l}\text { Ref } \\
J H K\end{array}$ & $\begin{array}{l}\text { Sp. } \\
\text { type }\end{array}$ & $\begin{array}{l}\text { Ref } \\
\text { SpT }\end{array}$ & GCVS & Var & $\begin{array}{l}P \\
\text { (d) }\end{array}$ & $K-[12]$ & Rem \\
\hline 35810 & 1686 & 0.38 & 1.26 & 4.18 & 9.01 & - & - & - & - & $\mathrm{R} 8$ & S28 & V758 Mon & - & & & \\
\hline 36623 & 1737 & 0.38 & 1.36 & 3.58 & 8.02 & 2.95 & 0.72 & 0.38 & N81 & R9 & $\mathrm{S} 28$ & NQ Gem & SR & 70 & 0.6 & $*$ \\
\hline 42975 & 2326 & 0.44 & 1.04 & 2.47 & 8.98 & 2.71 & 1.38 & 0.84 & W00a & $\mathrm{R}$ & S72 & R Pyx & Mira & 365 & 2.3 & \\
\hline 44812 & 2428 & 0.21 & 1.22 & 4.72 & 10.61 & 6.50 & 0.78 & 0.35 & $2 \mathrm{M}$ & R6 & S44 & & & & & \\
\hline 50412 & 2715 & 0.61 & 1.65 & 4.47 & 10.83 & 6.46 & 0.76 & 0.38 & $2 \mathrm{M}$ & $\mathrm{R} 6$ & S44 & & & & & \\
\hline 52656 & 2852 & 2.91 & 3.67 & 4.64 & 8.71 & 2.54 & 1.16 & 0.54 & K94 & $\mathrm{R}$ & K93 & TZ Car & SRb & 69 & 1.5 & \\
\hline 54806 & 2975 & - & - & - & 10.14 & - & - & - & - & R5 & $\mathrm{HD}$ & & & & & \\
\hline 60534 & 3227 & 1.19 & 1.84 & 2.85 & 7.52 & 2.53 & 0.95 & 0.43 & C79 & $\mathrm{R} 4$ & B96 & S Cen & SR & 65 & 1.3 & \\
\hline 62401 & 3286 & 0.24 & 1.67 & 11.75 & 11.98 & 1.81 & 1.85 & 1.3 & W00a & R3 & S44 & RU Vir & Mira & 433 & 4.1 & \\
\hline 66070 & 3405 & 0.37 & 1.08 & 3.20 & 8.51 & 3.52 & 0.94 & 0.37 & C79 & $\mathrm{R} 2$ & B96 & V971 Cen & - & - & 1.1 & \\
\hline 85750 & 3842 & 0.39 & 1.43 & 4.00 & 9.42 & 5.15 & - & - & D86 & $\mathrm{R} 2$ & V58 & & & & & \\
\hline 91929 & 4086 & 0.37 & 1.58 & 5.62 & 9.75 & - & - & - & - & $\mathrm{R} 4$ & B96 & RV Sct & $\mathrm{Lb}$ & - & - & \\
\hline 94049 & 4179 & - & - & - & 10.39 & - & - & - & - & $\mathrm{R} 4$ & $\mathrm{~S} 28$ & & & & & \\
\hline 95422 & 4263 & 0.37 & 1.45 & 5.69 & 11.01 & 6.74 & 0.75 & 0.26 & $2 \mathrm{M}$ & R5 & V58 & & & & & \\
\hline 98223 & 4567 & 0.83 & 2.25 & 6.10 & 9.36 & 5.15 & - & - & M65 & $\mathrm{R} 8$ & $\mathrm{~S} 28$ & & & & & \\
\hline 104522 & 5147 & 0.34 & 1.22 & 4.34 & 9.76 & 5.26 & 0.91 & 0.29 & C79 & R5 & $\mathrm{HD}$ & & & & 1.1 & \\
\hline 108205 & 5494 & 0.29 & 1.03 & 3.60 & 9.23 & 1.71 & - & - & IRC & $\mathrm{R} 2$ & V58 & LW Cyg & $\mathrm{Lb}$ & - & 1.5 & $*$ \\
\hline 109158 & 5577 & 0.46 & 1.55 & 5.25 & 10.12 & 2.57 & 1.21 & 0.75 & N81 & $\mathrm{R}$ & L44 & CT Lac & $\mathrm{SRa}$ & 555 & 2.4 & \\
\hline 113150 & 5761 & - & - & - & 10.75 & - & - & - & - & R5 & V58 & & & & & \\
\hline 113715 & 5791 & 0.77 & 1.69 & 3.72 & 9.66 & 2.99 & 1.02 & 0.82 & N81 & $\mathrm{R} 8$ & S44 & VY And & $\mathrm{SRb}$ & 149 & 1.7 & \\
\hline
\end{tabular}

\section{Notes:}

HIP 36623: also classified as symbiotic variable; HIP 108205: Li star (Boffin et al. 1993).

References to spectral types and magnitudes:

HD: Henry Draper Catalogue; IRC: Neugebauer \& Leighton (1969); 2M: Two Micron All-Sky Survey (Skrutskie 1997); B96: Barnbaum et al. (1996); C79: Catchpole et al. (1979); D86: Dominy et al. (1986); K93: Keenan (1993); K94: Kerschbaum \& Hron (1994); L44: Lee et al. (1944); M65: Mendoza V. \& Johnson (1965); N81: Noguchi et al. (1981); S28: Shane (1928); S44: Sanford (1944); S72: Stock \& Wroblewski (1972); V58: Vandervoort (1958); W00a: Whitelock et al. (2000).

are listed in Tables 1 and 2. Where available, we have listed the spectral type from R0 to R8.

Many of the remaining 317 carbon stars are spectroscopically classified only as carbon stars, and some of these may be $\mathrm{R}$ stars also. However, as the $(J-H, H-K)$ diagram shows (Fig. 1), not many non-R stars, apart from the $\mathrm{CH}$ stars, occupy the location of $\mathrm{R}$ stars in that diagram. $\mathrm{CH}$ stars must be treated separately from the $\mathrm{R}$ stars, as all $\mathrm{CH}$ stars are believed to be binary systems (McClure 1997a), and must therefore be processed with a binary astrometric model, as presented in Paper I (Pourbaix \& Jorissen 2000).

Further, since there is often disagreement in the various references as to the spectral type of a given star (examples were given in Sect. 2.1), the "spectral purity" of the sample of $\mathrm{R}$ stars collected in Tables 1 and 2 is best assessed by looking at the $(J-H, H-K)$ diagram.

The main source of confusion appears to be the fact that several late-type $\mathrm{R}$ stars from Table 2 are located in the region occupied by $\mathrm{N}$ stars. This is not totally surprising, as late $\mathrm{R}$ stars bear similarities with, or might even be identical to, $\mathrm{N}$ stars. In the following analysis, it will therefore be necessary to treat separately R stars with $H-K<0.3$ [or $(V-K)_{0}<4$; see Fig. 1] from those with $H-K \geq 0.3$. Finally, the few $\mathrm{R}$ stars also classified as $\mathrm{R}$ CrB variables should be treated separately as well.

To stress the fact that the early- and late-type $\mathrm{R}$ stars in the present sample must be considered separately, they have been split into two separate lists (Tables 1 and 2 respectively). The assignment of a star as an early- or latetype $\mathrm{R}$ star is mainly based on the $(V-K)_{0}$ index, with the threshold set at 4 . The $(V-K)_{0}$ index has preference over the $\mathrm{R}$ spectral subtype in case of conflicting classifications. When the $(V-K)_{0}$ index is not available, a criterion based on the spectral type is used (with the threshold set at R3.5). It will be shown below that, with this choice for the thresholds, the two subsamples of $\mathrm{R}$ stars present homogeneous properties with respect to the $R$ spectral subtype, to mass loss and to variability type, most late-type $\mathrm{R}$ stars being long-period variable stars, with circumstellar dust emission indicative of mass loss. The $K-[12]$ color index (with $K-[12]=K+2.5 \log F 12 / 28.3, F 12$ being the flux density in the IRAS $12 \mu \mathrm{m}$ band, expressed in $\mathrm{Jy}$ ) is considered as a good indicator of (dust) mass loss, 


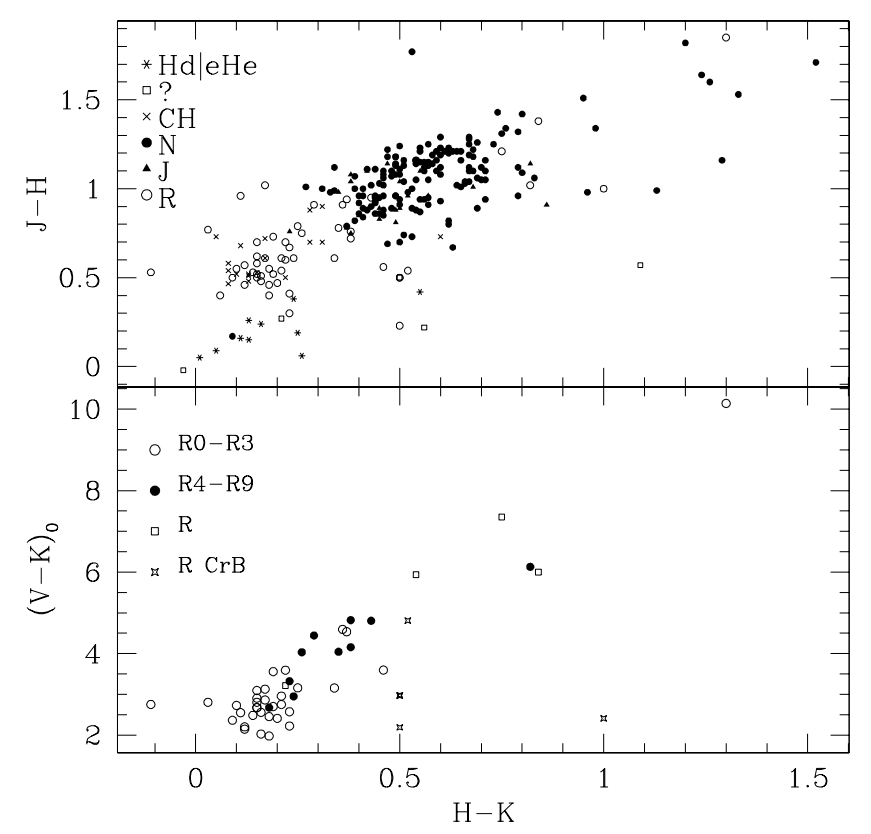

Fig. 1. Top panel: the $(J-H, H-K)$ color-color diagram for the carbon stars from the Hipparcos catalogue with $J, H$ and $K$ collected from the literature (as listed in Tables 1 and 2 for $\mathrm{R}$ stars). Symbols are as listed in the figure, with "Hd" and "eHe" standing for "hydrogen-deficient" and "extreme-helium" carbon stars. Hydrogen-deficient carbon stars are similar to $\mathrm{R}$ CrB carbon stars, except that they do not undergo obscuration episodes (Feast et al. 1997). Extreme-helium carbon stars are similar to, but hotter than, R CrB carbon stars (Cottrell \& Lawson 1998). Bottom panel: the $\left((V-K)_{0}, H-K\right)$ diagram for the sample of $\mathrm{R}$ stars considered in the present study

according to e.g., Fig. 21 of Jorissen \& Knapp (1998). Non-mass-losing stars have $K-[12] \sim 0.7$.

Late-type $\mathrm{R}$ stars are thus likely to belong to the masslosing AGB, and are therefore not considered further in the following discussion, which focuses on $\mathrm{R}$ stars with $(V-K)_{0}<4$.

\subsection{Parallaxes}

The Hipparcos catalogue (ESA 1997) gives parallax values at the $2 \sigma$ level or better [i.e. $\varpi / \epsilon(\varpi)>2$, where $\epsilon(\varpi)$ is the quoted parallax uncertainty] for $17 \mathrm{R}$ stars, of which 15 are of types $\mathrm{R} 0-\mathrm{R} 2$. The resulting absolute visual magnitudes for the early $\mathrm{R}$ stars cover a range of $M_{V}=-2.8$ to +4.1 (Knapp 2001). However, the parallax errors for many of the stars are larger, sometimes much larger, than the errors expected for the magnitudes and ecliptic coordinates of the stars. Of the nearly 320 carbon stars in the sample of Knapp (2001), only $25 \%$ (and only $18 \%$ of the $\mathrm{R}$ stars) have " $2 \sigma$ " parallaxes.

The Hipparcos Intermediate Astrometric Data (IAD; van Leeuwen \& Evans 1998) for the entire sample of carbon stars were therefore reprocessed as described and Papers I and III. Therefore, only a brief outline of the method is given here.
The position, distance modulus and proper motion are the five parameters in the model fit to be adjusted by $\chi^{2}$ minimization of the IAD abscissa residuals. Included in this fitting procedure is the rejection of outliers, defined as having a fit residual greater than three times the average of the residuals. In practice, two or three observations at most were rejected. Fitting the distance modulus ensures that the derived parallax $\varpi$ is always positive, as described in Paper I, but at the expense of asymmetric error bars and of a bias on $\varpi$. This bias is of any importance only when $\epsilon(\varpi) / \varpi \gtrsim 1$, as shown in Paper III. The distance modulus is better behaved, and must be used for computing average absolute magnitudes, as further discussed in Sect. 3.

Another major difference with respect to the reduction scheme applied by the Hipparcos consortia resides in the bootstrap algorithm used to reject those data points which yield inconsistent FAST and NDAC solutions, as described in detail in Paper III.

Monte-Carlo simulations have shown that this reprocessing scheme of the IAD fails when the noise on the $\mathrm{IAD}$ is too large with respect to the true parallax, in that it yields absolute magnitudes that are too faint by as much as 5 mag (i.e., parallaxes come out too large). This is not really unexpected, as the bootstrap method will remove "discrepant" observations until a solution passing through the remaining IAD is finally found. The parallax of that solution will clearly be of the order of the noise on the abscissa residuals, though the spurious nature of that solution may be noticed by the large uncertainty on the parallax. For the sample of $\mathrm{R}$ stars considered in the present paper, such a bias on the absolute magnitude becomes clearly apparent for stars with $K>8$, although the parallax of somewhat brighter stars is already biased as well, as seen in Fig. 2 (see also Sect. 3.1). In an attempt to keep as many unbiased stars as possible, the following criterion has been adopted to reject biased parallaxes: $K>8$ (or $V>10.5$ if no $K$ magnitude is available) or the star has a simulated absolute magnitude that lies further than $4 \sigma$ away from the true absolute magnitude of -2 on Fig. 2, $\sigma$ being the standard deviation of the simulated absolute magnitudes for stars with $K<7$ (i.e., which are not biased). Therefore, Tables 1 and 2 list only those parallaxes that may be considered as unbiased according to the above criteria.

The results are listed in Tables 1 and 2, which provides the HIP number from the Hipparcos catalogue in the first column. The star number in the General Catalog of Cool Galactic Carbon Stars (GCGCS; Stephenson 1989) is listed in Col. 2. Columns 3 to 5 provide the parallax $\varpi_{0}$ derived from the $\chi^{2}$ minimization on the distance modulus, the corresponding lower and upper bounds $\left(\varpi_{\mathrm{L}}\right.$ and $\left.\varpi_{\mathrm{U}}\right)$ computed from Eq. (9) of Paper I. The following columns list the apparent $V$ magnitude (field $\mathrm{H} 5$ of the Hipparcos catalogue), the $K$ magnitude, the $J-H$ and $H-K$ color indices, their reference, and finally the spectral type and its reference. 


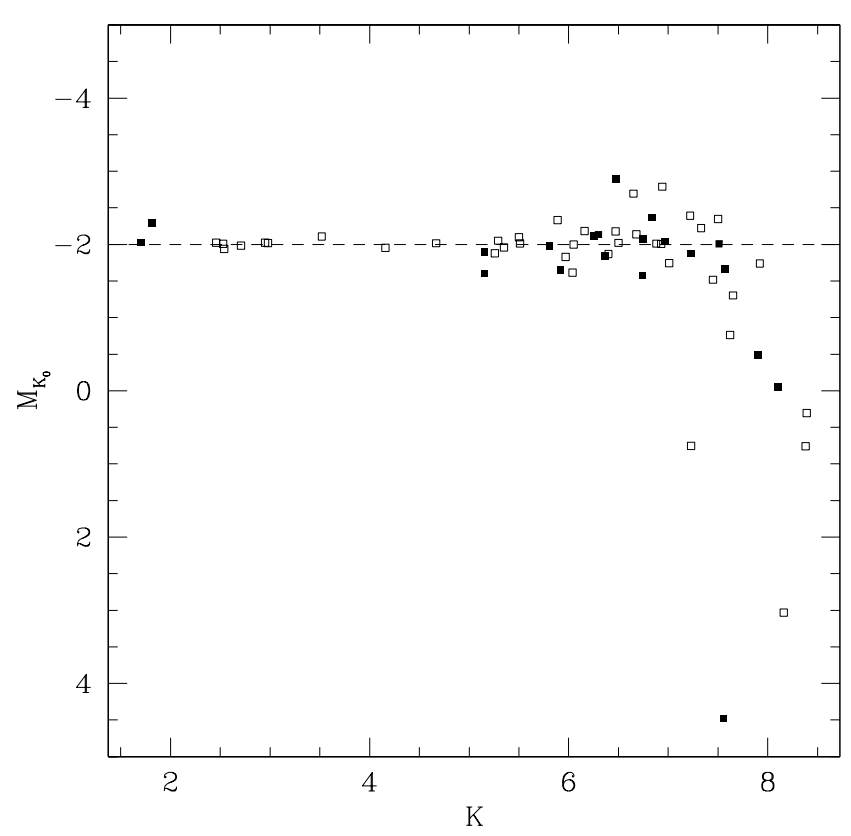

Fig. 2. The absolute magnitudes $M_{K}$ retrieved by processing the IAD as indicated in Sect. 2.5 for the sample stars assuming that they all have a true absolute magnitude $M_{K}^{0}=-2.0$ (see also Sect. 3.1). It is clearly seen that a strong bias on $M_{K}$ appears for stars with $K \gtrsim 8$ that have noisy IAD. Stars with negative parallaxes in the Hipparcos catalogue are identified by filled squares

\section{The absolute magnitudes and colors of $\mathbf{R}$ stars}

The absolute magnitudes $M_{V}$ and $M_{K}$ are calculated as follows:

$d=1 / \varpi\left(^{\prime \prime}\right)$

$M_{X}=m_{X}+5-5 \log d-A_{X} \quad($ where $X=V$ or $K)(2)$

$A_{K}=0.114 A_{V}$

where $d$ is the distance in pc, and $A_{V}$ and $A_{K}$ are the interstellar absorptions in the $V$ and $K$ bands, respectively. We have adopted the extinction model of Hakkila et al. (1997) with the $A_{K} / A_{V}$ ratio from Cardelli et al. (1989). We do not attempt to include circumstellar extinction, since this is shown by infrared color to be small or nonexistent for the $\mathrm{R}$ stars. The extinction corrections to the $K$ magnitudes are small, less than $0.2 \mathrm{mag}$.

Figure 3 shows the color - absolute magnitude diagram, $M_{K_{0}}$ versus $(V-K)_{0}$, for the stars with available $K$ magnitudes. The error bars on $M_{K}$ are calculated from the upper and lower bounds on the parallax listed in Tables 1 and 2. As noted in Paper I, the parallax uncertainties are computed so as to produce symmetric error bars on the magnitude. The resulting uncertainties in the absolute magnitudes are often larger than 1 mag (see Fig. 3), and dwarf the uncertainties on the measured apparent magnitudes and on the interstellar extinction.

Figure 3 shows that the difference between late- and early-type R stars, assessed from their photometric, spectral, variability and mass-loss properties as discussed in

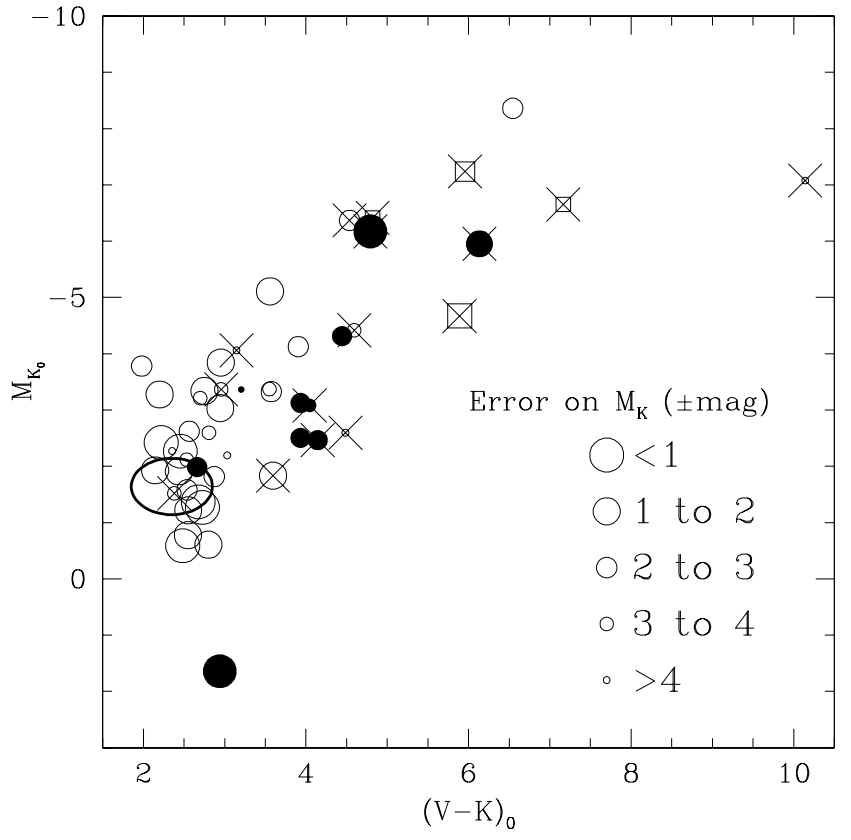

Fig. 3. Dereddened absolute magnitude $M_{K_{0}}$ versus $(V-K)_{0}$ color for R stars. Open symbols: stars of spectral type R0-R3; filled symbols: stars of type R4-R8; squares: stars of unknown $\mathrm{R}$ subtype. The symbol size is inversely proportional to the error on $M_{K}$, as calculated from the upper and lower bounds on the parallax listed in Tables 1 and 2. Large crosses denote stars with $H-K>0.3$ that are more likely to be $\mathrm{N}$-type rather than R-type carbon stars (see Fig. 1). Only stars with $K \leq 8$ have been included. The ellipse indicates the average location of clump giants, according to Alves (2000, see also Fig. 5)

Sect. 2.4, is also apparent in their luminosities: late-type $\mathrm{R}$ stars with $(V-K)_{0}>4$ tend to be more luminous than early-type $\mathrm{R}$ stars. Late-type $\mathrm{R}$ stars thus clearly mark the bottom of the asymptotic giant branch.

\subsection{Average absolute magnitude of early-type $R$ stars}

To derive an average absolute magnitude from a set of measured parallaxes is an operation plagued by many biases (e.g., Arenou \& Luri 1999), and several simulations have been performed to identify their importance for the present sample.

More precisely, the simulations described below evaluate whether the reduction scheme used in the present work is able to correctly retrieve the absolute magnitude of the sample stars. In a first step, it is assumed that all the 59 stars from the present sample with a $K$ magnitude available have the same absolute magnitude, namely $M_{K}^{0}=-2.0$. In that framework, a true parallax may be assigned to each star of the sample, depending on its observed apparent $K$ magnitude according to the relation $\log \varpi=0.2\left(M_{K}^{0}-K\right)-1$. In the first simulation, the original abscissa residuals of the IAD file were replaced by residuals drawn from a normal distribution of zero mean and $\sigma_{\mathrm{v}}=1$ mas (standard deviation of the residuals). To artificially increase the sample size, 32 different sets 


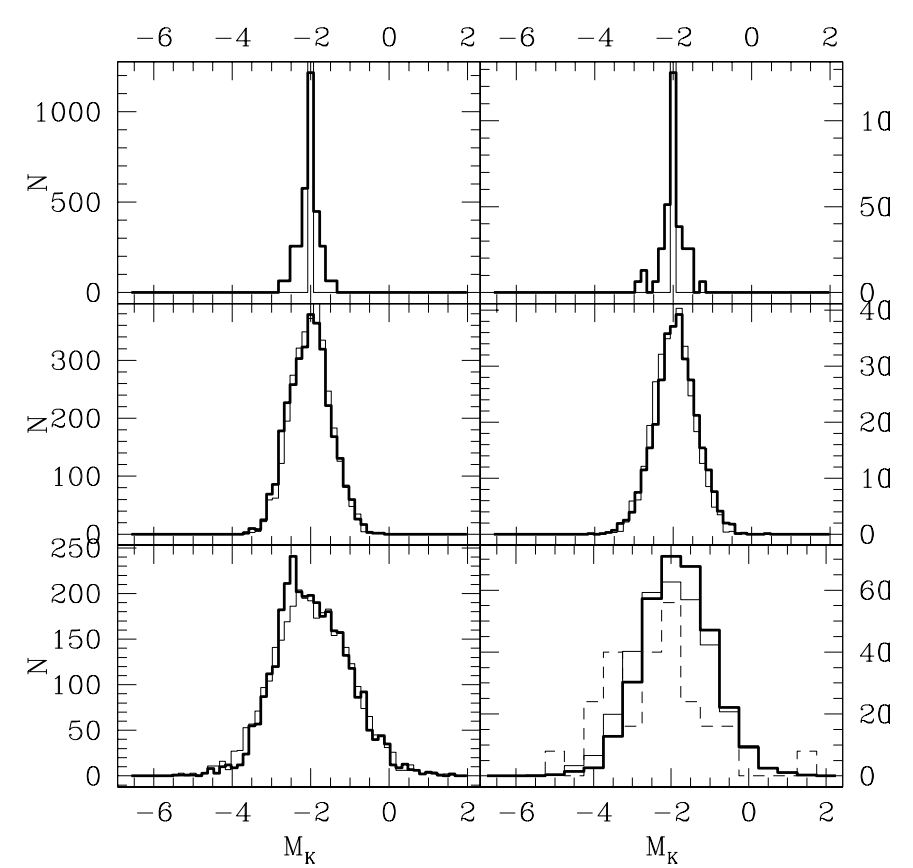

Fig. 4. The distribution of absolute magnitudes $M_{K}$ for the sample of $\mathrm{R}$ stars with $K \leq 8$ and $(V-K)_{0}<4$ obtained by reprocessing the IAD as indicated in Sect. 2.5 (thick line), for different assumptions of the true $M_{K}$ distribution (thin line): upper panel: $M_{K}^{0}=-2.0$ for all stars; (ii) middle panel: Gaussian distribution of mean $M_{K}^{0}=-2.0$ and $\sigma_{0}=0.5 \mathrm{mag}$; (iii) bottom panel: Gaussian distribution of mean $M_{K}^{0}=-2.0$ and $\sigma_{0}=1.0 \mathrm{mag}$. The left panels correspond to the simulations performed on the IAD files with abscissa residuals drawn from a normal distribution of mean zero and $\sigma_{\mathrm{v}}=1$ mas, while the right panels correspond to the simulations performed on the IAD files with the actual abscissa residuals. In the bottom right panel, the actual observed distribution (scaled by a factor of 30) is represented by the dashed line. The bin size in that panel has been enlarged due to the smaller size of the observed star sample

of residuals are drawn for the IAD file corresponding to each of the $59 \mathrm{R}$ stars, totaling to 1888 simulated stars. In this test, the IAD file only serves to define the time sampling of the observations and the scanning angle on the sky for each observation. The reduction scheme outlined in Sect. 2.5 is then applied to the total sets of modified IAD files. Results are shown in the upper left panel of Fig. 4. In a second simulation, the reduction scheme is applied to the actual abscissa residuals which do not necessarily follow a normal distribution with a standard deviation of $\sigma_{\mathrm{v}}=1$ mas. Any differences between the results of the first and second simulation will thus highlight the impact of the measurement errors.

The results are shown in the upper panel of Fig. 4, and the first three moments of the retrieved distributions are listed in Table 3, where the coefficient of skewness is defined as $\mu_{3} / \sigma^{3}$ (and $\mu_{3}$ is the centered moment of order 3 ). The method is able to retrieve the true absolute magnitude when the uncertainties on the residuals have a standard deviation of 1 mas. If the actual residuals are used instead, the computed distribution is retrieved as well, except for a tail extending towards fainter $M_{\mathrm{K}}$, as apparent in Fig. 2. That tail is not visible in Fig. 4, though, since stars with $K>8$ have very large and noisy abscissa residuals and were not displayed.

Other simulations were carried out as above, except that Gaussian distributions with $\sigma_{0}=0.5$ and $1 \mathrm{mag}$ were adopted for the true absolute magnitude distribution. Basically the same features as for the case $\sigma_{0}=0$ emerge, namely the fact that the retrieved distribution using the actual IAD residuals is skewed towards fainter absolute magnitudes, with a bias of $0.55 \mathrm{mag}$ for the $\sigma_{0}=1 \mathrm{mag}$ case, which however reduces to 0.16 mag if only stars with $K \leq 8$ are kept (Table 3 ).

The distribution derived from the true distribution $\left(\overline{M_{K}^{0}}, \sigma_{0}\right)=(-2.0,1.0)$ provides a fair match to the observed distribution (bottom right panel of Fig. 4), characterized by a weighted-average absolute magnitude

$\overline{M_{K}}=\frac{\sum_{i=1}^{N} M_{K, i} w_{i}}{\sum_{i=1}^{N} w_{i}}=-1.54$,

where $M_{K, i}$ is the individual absolute magnitude of $\mathrm{R}$ stars with $(V-K)_{0}<4$ and $K \leq 8, w_{i}=1 / \epsilon\left(M_{K, i}\right)^{2}$ and $\epsilon\left(M_{K, i}\right)=5\left(\log \varpi_{\mathrm{U}}-\log \varpi_{0}\right)=5\left(\log \varpi_{0}-\log \varpi_{\mathrm{L}}\right)$, and $\varpi_{0}, \varpi_{\mathrm{L}}, \varpi_{\mathrm{U}}$ as derived by our reduction scheme are given in Tables 1 and 2 .

Thus it may be concluded that the early-type $R$ stars have a true absolute-magnitude distribution close to $\left(\overline{M_{K}^{0}}, \sigma_{0}\right)=(-2.0,1.0)$. Since the present sample of $\mathrm{R}$ stars is basically magnitude-limited, it is subject to the Malmquist bias favoring high-luminosity stars. The Malmquist (1936) bias is probably responsible for the high-luminosity tail and the deficit of low-luminosity stars in the observed sample (bottom-right panel of Fig. 4), as compared to the simulated Gaussian population.

\subsection{Early $R$ stars as members of the red clump}

Figure 5 reproduces the color-magnitude diagram from Fig. 3 for $\mathrm{R}$ stars with $(V-K)_{0}<4$ and $K \leq 8$, and compares them to 238 nearby red clump stars as compiled by Alves (2000). The error bars have been excluded for clarity. Red clump stars have become of renewed interest recently because Hipparcos has made possible the calibration of their absolute magnitudes via observations of nearby stars (Paczyński \& Stanek 1998; Girardi et al. 1998), making them a powerful tool for distance measurements within the Galaxy and the Local Group. Physically, these objects are the analogues of the red horizontal branch stars seen in metal-rich globular clusters: helium core burning post-red-giant branch stars. Figure 5 very strongly suggests that the $R$ stars are members of the red clump, since the inferred true absolute magnitude $M_{K}^{0}=-2.0$ (with an estimated intrinsic dispersion of $1.0 \mathrm{mag}$ ) of $\mathrm{R}$ stars (Sect. 3.1) is similar to the average $M_{K}=-1.61 \pm 0.03$ derived from the sample of Alves (2000). The $V-K$ colors of $\mathrm{R}$ stars show a somewhat broader spread (but this may be due to contamination by a few $\mathrm{N}$ stars), and they are on average about 0.5 mag redder 
Table 3. Weighted average, standard deviation and coefficient of skewness of the retrieved $M_{K}$ distributions for different choices of the "true" $\left(\overline{M_{K}^{0}}, \sigma_{0}\right)$ distributions and input IAD files (see text)

\begin{tabular}{|c|c|c|c|c|c|c|}
\hline \multirow[t]{2}{*}{ IAD abscissa residuals: } & \multicolumn{3}{|c|}{ Gaussian with $\sigma_{\mathrm{v}}=1$ mas } & \multicolumn{3}{|c|}{ actual } \\
\hline & $\overline{M_{K}}$ & $\sigma$ & skewness & $\overline{M_{K}}$ & $\sigma$ & skewness \\
\hline \multicolumn{7}{|l|}{ True distribution: } \\
\hline$\overline{M_{K}^{0}}=-2.0, \sigma_{0}=0.0 \mathrm{mag}$ & -2.01 & 0.29 & 0.09 & -1.61 & 1.24 & 3.15 \\
\hline$\overline{M_{K}^{0}}=-2.0, \sigma_{0}=0.5 \mathrm{mag}$ & -1.98 & 0.54 & 0.01 & -1.53 & 1.29 & 2.59 \\
\hline$\overline{M_{K}^{0}}=-2.0, \sigma_{0}=1.0 \mathrm{mag}$ & -1.89 & 0.91 & 0.16 & -1.45 & 1.53 & 1.63 \\
\hline
\end{tabular}

\begin{tabular}{|c|c|c|c|c|c|c|}
\hline \multirow[t]{2}{*}{ IAD abscissa residuals: } & \multicolumn{3}{|c|}{ Gaussian with $\sigma_{\mathrm{v}}=1$ mas } & \multicolumn{3}{|c|}{ actual } \\
\hline & $\overline{M_{K}}$ & $\sigma$ & skewness & $\overline{M_{K}}$ & $\sigma$ & skewness \\
\hline \multicolumn{7}{|l|}{ True distribution: } \\
\hline$\overline{M_{K}^{0}}=-2.0, \sigma_{0}=0.0 \mathrm{mag}$ & -2.05 & 0.24 & -0.24 & -2.02 & 0.29 & -0.58 \\
\hline$\overline{M_{K}^{0}}=-2.0, \sigma_{0}=0.5 \mathrm{mag}$ & -2.03 & 0.53 & 0.07 & -1.93 & 0.54 & -0.05 \\
\hline$\frac{\kappa}{M_{K}^{0}}=-2.0, \sigma_{0}=1.0 \mathrm{mag}$ & -1.95 & 0.93 & 0.20 & -1.84 & 0.90 & 0.03 \\
\hline
\end{tabular}

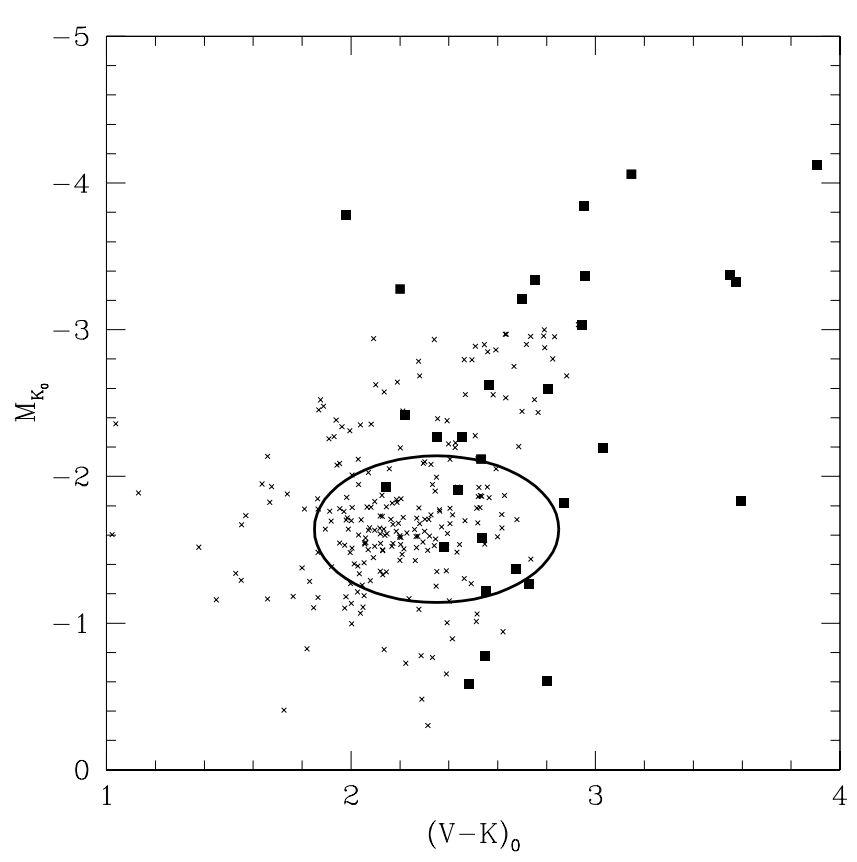

Fig. 5. $M_{K}$ vs. $V-K$ for early $\mathrm{R}$ stars with $K \leq 8$ (filled symbols), and red clump giants (squares) from Alves (2000). The ellipse is the same as in Fig. 3

than the local clump stars, which have $\langle V-K\rangle=2.35$; this is likely to be due to the presence of weak to moderate $\mathrm{C}_{2}$ and $\mathrm{CN}$ band absorption in the blue region of the spectrum.

\section{The space density of early $R$ stars}

In this section, the space density of $\mathrm{R}$ stars will be estimated and compared to that of red clump stars. To evaluate the completeness of the sample of early $\mathrm{R}$ stars, Fig. 6 displays the cumulative frequency distribution of early $\mathrm{R}$ stars as a function of distance. To circumvent the bias on the derived absolute magnitude distribution (see Fig. 4), the distances have been estimated by assigning the absolute magnitude $M_{K}^{0}=-2.0$ to all early $\mathrm{R}$ stars. Under the assumption of a uniform density $n_{0}$ in the plane and an exponential distribution perpendicular to the plane with scale height $z_{0}$, the cumulative frequency distribution varies with distance $d$ as

$\log N(d)=4 \pi n_{0} d^{3} \times$

$\left[\left(\frac{1}{2} \frac{z_{0}}{d}-\left(\frac{z_{0}}{d}\right)^{3}\right)+\left(\left(\frac{z_{0}}{d}\right)^{2}+\left(\frac{z_{0}}{d}\right)^{3}\right) \exp \left(-\frac{d}{z_{0}}\right)\right]$

The scale height and the space density of $\mathrm{R}$ stars can then be estimated from the values that must be assigned to the parameters $z_{0}$ and $n_{0}$ in order to fit the observed distribution. It is found to be about $n_{0} \sim 4.510^{-8} \mathrm{pc}^{-3}$ for $z_{0}=300 \mathrm{pc}$. Figure 6 reveals that, for this parameter set, early R stars follow the predicted uniform/exponential distribution up to about $600 \mathrm{pc}$ (involving about $60 \%$ of the total sample). It needs not be emphasized that these values are very uncertain though, as $z_{0}$ is only poorly constrained by the fit and both $z_{0}$ and $n_{0}$ depend on the quite uncertain $M_{K}^{0}$ assignment. Adopting $M_{K}^{0}=-1$ instead yields $n_{0} \sim 1.510^{-7} \mathrm{pc}^{-3}$ and $z_{0} \sim 180 \mathrm{pc}$.

The sample of red clump stars by Alves (2000) contains 284 stars with $5 \%$ parallaxes from the Hipparcos catalogue and is roughly complete to 12 mas or about $83 \mathrm{pc}$. The volume density of the red clump stars is thus about $1.1410^{-4} \mathrm{pc}^{-3}$ and the $\mathrm{R}$ stars form about $0.04 \%$ (or $0.13 \%$ if $M_{K}^{0}=-1$ ) of the total. There are additional uncertainties because, for a given star, there is no way to tell whether it is a clump star or is on the red giant branch. Nevertheless, the qualitative conclusion is clear: a very small fraction of clump stars in the solar neighborhood is carbon-rich. 


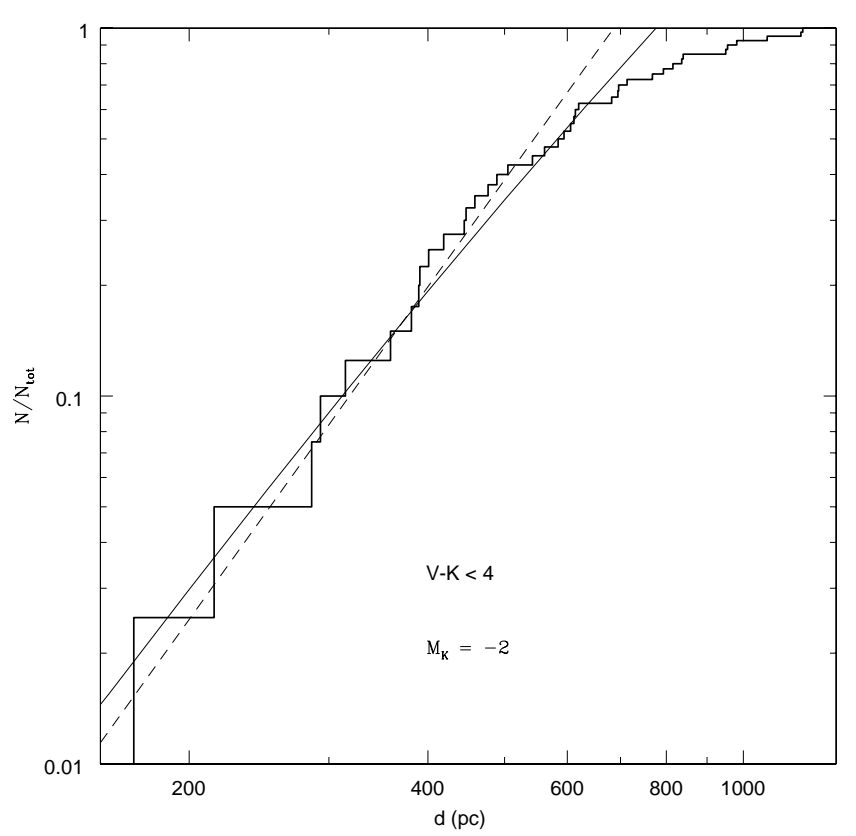

Fig. 6. Cumulative frequency distribution of the distances of early R stars (thick line), as compared to predictions for a stellar sample uniformly distributed in the plane and with an exponential distribution perpendicular to the plane (thin line). A scale height of $300 \mathrm{pc}$ and a true absolute magnitude $M_{K}^{0}=-2.0$ have been adopted. The thin dashed line corresponds to the predictions for a spherically-symmetric homogeneous sample

\section{Conclusions}

In this paper, we have re-analyzed the Hipparcos Intermediate Astrometric Data for a sample of 83 carbon stars of class R, the class of warm evolved carbon red giants. A special reprocessing scheme has been used, fitting the distance modulus instead of the parallax (thus ensuring its positiveness) and rejecting outliers within a bootstrap scheme ensuring consistency between the solutions obtained from the NDAC and FAST data. This reduction scheme is not free from biases: Monte-Carlo simulations have shown that it tends to produce parallaxes that are too large when $\epsilon(\varpi) / \varpi \gtrsim 1$. Despite these biases, the simulations allow the identification of the true properties of the population.

We have shown that the early- and late-type $\mathrm{R}$ stars have different properties. Late-type $\mathrm{R}$ stars generally have subtypes later than R4, although a better definition uses their color indices $(V-K)_{0}>4$ or $(H-K)>0.3$. Most of these stars are long-period variables of the SR or Mira types, and they often exhibit excess emission at $12 \mu \mathrm{m}$ due to dust, indicative of mass loss. In the HR diagram, these stars mark the bottom of the AGB, and are thus related, or even identical, to the $\mathrm{N}$ stars.

The early-type $\mathrm{R}$ stars form a rather homogeneous group, with most of its members populating the red clump. They are well represented by a population having a Gaussian distribution of absolute magnitudes such that $\left(M_{K}^{0}, \sigma_{0}\right)=(-2 ., 1$.$) . They are not variable and do$ not appear to suffer from substantial mass loss, as judged from the absence of $12 \mu \mathrm{m}$ excess. They represent fewer than $0.1 \%$ of the clump stars. Their nature as carbon stars is not due to low metallicity: $\mathrm{R}$ stars have metal abundances which are close to solar (Dominy 1984), except for the carbon excess.

The location of the $\mathrm{R}$ stars in the red clump provides strong support for the formation scenario suggested by Dominy (1984): the helium core flash mixes carbon to the surface of the star. This mixing episode may perhaps be rotationally-induced as the star is spun up by the accretion of a former companion. This scenario has the advantage of accounting as well for the lack of binary systems among R stars (McClure 1997b), but it requires a braking mechanism (e.g. magnetic braking as advocated by Habets \& Zwaan 1989) since R stars do not currently appear to be rapid rotators. The fraction of stars which become $\mathrm{R}$ stars is much smaller than the fraction $(10 \%-50 \%$, see Wallerstein \& Knapp 1998) of stars which become carbon stars on the AGB, and it is unlikely to have any significant influence on the number of carbon AGB stars. It suggests that a small fraction of the RR Lyrae stars and blue horizontal branch stars in the Galactic halo are also carbon rich - these stars however are too hot to have $\mathrm{C}_{2}$ in the atmosphere.

Acknowledgements. We thank Bohdan Paczyński and Jim Gunn for useful discussions and advice, David Alves for a machine-readable copy of his red clump star data, and the National Aeronautics and Space Administration NASA who supported this work via grants NAG5-6734 and NAG5-8083. We also thank Frédéric Arenou, the referee, for his useful comments and suggestions. This publication makes use of data products from the Two Micron All Sky Survey, which is a joint project of the University of Massachusetts and the Infrared Processing and Analysis Center/California Institute of Technology, funded by the National Aeronautics and Space Administration and the National Science Foundation. This research has made use of the Simbad data base, operating at CDS, Strasbourg, France.

\section{References}

Alves, D. R. 2000, ApJ, 539, 732

Arenou, F., \& Luri, X. 1999, in Harmonizing Cosmic Distance Scales in a Post-Hipparcos Era ASP Conference Series \#167, ed. D. Egret, \& A. Heck

Barnbaum, C., Stone, R. P. S., \& Keenan, P. C. 1996, ApJS, 105, 419

Bartkevičius, A. 1996, Baltic Astron., 5, 217

Boffin, H. M. J., Abia, C., Isern, J., \& Rebolo, R. 1993, A\&AS, 102,361

Bond, H. E. 1975, ApJ, 202, L47

Cannon, A. J. 1921, Circ. Harvard Obs., 224

Cardelli, J. A., Clayton, G. C., \& Mathis, J. S. 1989, ApJ, 345, 245

Catchpole, R. W., Robertson, B. S. C., Lloyd Evans, T. H. H., et al. 1979, S. Afr. Obs. Circ., 1, 61

Cohen, J. G., Frogel, J. A., Persson, S. E., \& Elias, J. H. 1981, ApJ, 249, 481 
Cottrell, P. L., \& Lawson, W. A. 1998, Publ. Astron. Soc. Aust., 15, 179

Dahn, C. C., Liebert, J., Kron, R. G., Spinrad, H., \& Hintzen, P. M. 1977, ApJ, 216, 757

Dean, C. A. 1976, AJ, 81, 364

Dominy, J. F. 1984, ApJS, 55, 27

Dominy, J. F., Lambert, D. L., Gehrz, R. D., \& Mozurkewich, D. 1986, AJ, 91, 951

Elias, J. H. 1978, AJ, 83, 791

ESA 1997, The Hipparcos and Tycho Catalogues (ESA SP-1200)

Feast, M. W., Carter, B. S., Roberts, G., Marang, F., \& Catchpole, R. M. 1997, MNRAS, 285, 317

Feast, M. W., \& Glass, I. S. 1973, MNRAS, 161, 293

Feast, M. W., \& Whitelock, P. A. 1992, MNRAS, 259, 6

Gezari, D. Y., Pitts, P. S., \& Schmitz, M. 1999, Catalog of Infrared Observations, 5th edn.

Girardi, L., Groenewegen, M. A. T., Weiss, A., \& Salaris, M. 1998, MNRAS, 301, 149

Green, P. J., \& Margon, B. 1994, ApJ, 423, 723

Habets, G. M. H. J., \& Zwaan, C. 1989, A\&A, 211, 56

Hakkila, J., Myers, J. M., Stidham, B. J., \& Hartmann, D. H. 1997, AJ, 114, 2042

Iben, Jr., I., \& Renzini, A. 1983, ARA\&A, 21, 271

Jorissen, A., \& Knapp, G. R. 1998, A\&AS, 129, 363

Jorissen, A., Van Eck, S., Mayor, M., \& Udry, S. 1998, A\&A, 332,877

Keenan, P. C. 1942, ApJ, 96, 101

Keenan, P. C. 1993, PASP, 105, 905

Kerschbaum, F., \& Hron, J. 1994, A\&AS, 106, 397

Kholopov, P. N., Samus, N. N., Frolov, M. S., et al. 1998, Combined General Catalogue of Variable Stars, 4th edn.

Knapp, G. R. 2001, ApJ, submitted

Lee, O. J., Gore, G. D., \& Bartlett, T. J. 1944, Annals Dearborn Obs., 5, 1

Malmquist, K. G. 1936, Meddel. Stockholm Obs., 26
Mayall, M. M. 1951, Bull. Harvard Obs., 920, 32

McClure, R. D. 1984a, PASP, 96, 117

McClure, R. D. 1984b, ApJ, 280, L31

McClure, R. D. 1997a, PASP, 109, 536

McClure, R. D. 1997b, PASP, 109, 256

Mendoza V., E. E., \& Johnson, H. L. 1965, ApJ, 141, 161

Neugebauer, G. \& Leighton, R. B. 1969, Two-Micro Sky Survey Catalogue (NASA SP-3047)

Noguchi, K., Kawara, K., Kobayashi, Y., et al. 1981, PASJ, 33, 373

Ohnaka, K., \& Tsuji, T. 1999, A\&A, 345, 233

Paczyński, B., \& Stanek, K. Z. 1998, ApJ, 494, L219

Pickering, E. C. 1896, Harvard Circ.

Pickering, E. C. 1908, Harvard Circ.

Pourbaix, D., \& Jorissen, A. 2000, A\&AS, 145, 161

Pourbaix, D., Knapp, G. R., \& Jorissen, A. 2001a, A\&A, in preparation

Pourbaix, D., Knapp, G. R., \& Jorissen, A. 2001b, A\&A, in preparation

Sanford, R. F. 1944, ApJ, 99, 145

Scalo, J. M. 1976, ApJ, 206, 474

Shane, C. D. 1928, Lick Obs. Bull., 13, 123

Skrutskie, M. 1997, S\&T, 94, 46

Slettebak, A., Keenan, P. C., \& Brundage, R. K. 1969, AJ, 74, 373

Stephenson, C. B. 1989, Publ. Warner \& Swasey Obs., 3, 1

Stock, J., \& Wroblewski, H. 1972, Publ. Dept. Astron. Univ. Chile, 2, 1

Ulla, A., Thejll, P., Kipper, T., \& Jorgensen, U. G. 1997, A\&A, 319,244

van Leeuwen, F., \& Evans, D. W. 1998, A\&AS, 130, 157

Vandervoort, G. L. 1958, AJ, 63, 477

Wallerstein, G. \& Knapp, G. K. 1998, ARA\&A, 36, 369

Whitelock, P., Marang, F., \& Feast, M. 2000, MNRAS, submitted

Yamashita, Y. 1975, PASJ, 27, 325 